\title{
Dose findings of antofloxacin hydrochloride for treating bacterial infections in an early clinical trial using PK-PD parameters in healthy volunteers
}

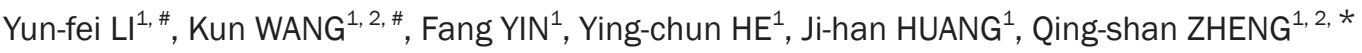 \\ ${ }^{1}$ Center for Drug Clinical Research, Shanghai University of Chinese Medicine, Shanghai 201203, China; ${ }^{2}$ Shanghai Municipal Educa- \\ tion Commission, E-Institute of Traditional Chinese Internal Medicine, Shanghai University of Traditional Chinese Medicine, Shanghai \\ 201203, China
}

Aim: To find an appropriate dose regimen of the novel antibacterial agent antofloxacin for a phase II clinical trial using a population pharmacokinetic (PPK) study in healthy volunteers and the minimum inhibitory concentration (MIC) as pharmacodynamic (PD) parameters.

Methods: Twenty-four healthy volunteers were enrolled in a double-blind crossover study and received antofloxacin (200 or $400 \mathrm{mg} / \mathrm{d}$, po) for consecutive $5 \mathrm{~d}$ with $10 \mathrm{~d}$ washout between two separate periods. Blood concentrations were analyzed using HPLC with a UVVis detector. The values of area under the curve (AUC) with covariates were obtained from a PPK model, and the MICs came from the previous in vitro studies. The dose regimen was determined for the phase II clinical trial according to the ratio (>20) of AUC/MIC, and the efficacy of the dose was evaluated by the trial.

Results: A two-compartment model best described the time-concentration data with first-order absorption. The PPK parameter estimates for $\mathrm{CL}, V_{c}, Q, V_{p}$ and $K_{A}$ are $8.34 \mathrm{~L} / \mathrm{h}, 142 \mathrm{~L}, 15.9 \mathrm{~L} / \mathrm{h}, 52.2 \mathrm{~L}$ and 4.64 1/h, respectively. The covariates sex for $K_{\mathrm{A}}$, weight for $\mathrm{CL}$, weight for $V_{c}$ and interoccasion variability were included in the final model. The AUC/MIC was calculated based on the PPK model and the MIC of antofloxacin for Escherichia coli, Klebsiella pneumonia, Staphylococcus aureus and Staphylococcus epidermidis were determined in previous researches. The $400 \mathrm{mg}$ loading dose with $200 \mathrm{mg} / \mathrm{d}$ maintenance dose was recommended and confirmed by the phase II trial.

Conclusion: The ratio of AUC from the PPK model vs MIC as the PD parameter can be applied in a dose-finding trial of antofloxacin in treatment of bacterial infections. The PPK model suggests that sex and body weight may be considerations in regards to individual therapy, which should be investigated in larger clinical trials and serve as a potential reference for clinical therapies.

Keywords: antofloxacin; phase II clinical trial; dosing regimens; population pharmacokinetics; AUC; MIC; Escherichia coli; Klebsiella pneumonia; Staphylococcus aureus; Staphylococcus epidermidis

Acta Pharmacologica Sinica (2012) 33: 1424-1430; doi: 10.1038/aps.2012.68; published online 6 Aug 2012

\section{Introduction}

A new fluoroquinolone compound developed by Anhui Huanqiu Pharmaceutical Company, antofloxacin (ATFX), is an 8-amino derivative of levofloxacin with potent antibacterial activities both in vitro and in vivo. Compared to some conventional quinolones such as ciprofloxacin, ofloxacin, and sparfloxacin, ATFX exhibits good antibacterial activity against quinolone-resistant, methicillin-resistant Staphylococcus aureus and Staphylococcus epidermidis ${ }^{[1]}$.

\footnotetext{
\# The two authors contributed equally to this work.

* To whom correspondence should be addressed.

E-mail zhengqscn@21cn.com

Received 2012-02-09 Accepted 2012-05-09
}

Certain demographic and pathophysiological features of patients such as body weight, excretory and metabolic functions, and concomitant therapy, may regularly alter dose-concentration relationships. Population pharmacokinetics (PPK) can be used to describe and predict an individual pharmacokinetic feature. It can also help identify the difference among population subgroups and determine the source and correlates of variability in drug concentrations among individuals in the target patient population receiving a clinically relevant dose of a drug of interest ${ }^{[2]}$. Although the pharmacokinetics of ATFX in healthy male volunteers has been reported ${ }^{[3-6]}$, it is not clear whether individual differences affect the PK characteristics in the Chinese population.

In addition, parameters such as $\mathrm{AUC} / \mathrm{MIC}, \mathrm{C}_{\max } / \mathrm{MIC}$, and 
$\mathrm{T}>\mathrm{MIC}$ have been used as effective indicators of antibacterial efficacy, as they are closely related to clinical outcomes ${ }^{[7]}$. The antimicrobials can usually be classified into two categories: time-dependent antimicrobials, such as $\beta$-lactams and macrolides, and concentration-dependent antimicrobials, such as fluoroquinolones ${ }^{[7]}$. AUC/MIC has usually been identified as an important pharmacodynamics parameter for fluoroquinolones when evaluating quinolone efficacy ${ }^{[8]}$. The minimum inhibitory concentration (MIC) for a specific bacterium is usually constant; AUC is therefore a pivotal factor in dose findings. In clinical treatment, a loading dose following a maintenance dose is often applied for the purpose of reaching or exceeding the steady-state concentration (Css) rapidly, especially when treating infections ${ }^{[9]}$. In the present study, we applied ATFX blood concentrations from a PK study in healthy volunteers to build a PPK model with consideration of the interoccasion variability (IOV). Depending on the AUC from the model and the clinical MIC measurement, the efficacies of different doses, with or without a loading dose, can be simulated and predicted. This may be regarded as a reference for designing a dose regimen for subsequent clinical trials.

\section{Materials and methods Patients}

This study was conducted at the Institute of Clinical Pharmacology of Peking University First Hospital, China. All volunteers were 20-40 years old with a body mass index (BMI) of 17-26 [BMI=body weight $(\mathrm{kg}) /$ height $\left.^{2}\left(\mathrm{~m}^{2}\right)\right]$. Laboratory tests showed that all participants were normal and healthy and did not have any organic diseases. The exclusion criteria included the following: (1) use of antibiotics, nonsteroidal antiinflammatory drugs, diuretics, antacids containing aluminum or magnesium, or anticoagulant in the past two weeks; (2) drug dependence, alcoholism or smoking currently or previously; and (3) fever in the past three days. The study was approved by the Independent Ethics Committee, and informed consent was obtained from each volunteer before enrollment. The investigational drug was provided by Anhui Huanqiu Pharmaceutical Company.

\section{Study design}

Twenty-four volunteers (12 men and 12 women) were recruited for this study. Each subject received 200 and 400 $\mathrm{mg} / \mathrm{d}$ of ATFX orally during two separate periods in the crossover study. The drug administration lasted for $5 \mathrm{~d}$ with a subsequent $10-\mathrm{d}$ washout. Blood samples were collected at $0.5,1,2,3,4,6,8,12,24$, and $48 \mathrm{~h}$ after administration on $\mathrm{d} 1$ and $\mathrm{d} 5$ of each period. Samples for the trough concentration were collected before dosing on d 2-d 4 of each period. Serum samples were isolated from blood samples and stored at $-20^{\circ} \mathrm{C}$.

\section{Determination of blood concentrations of antofloxacin}

To a $500 \mu \mathrm{L}$ serum sample, $200 \mu \mathrm{L}$ of $10 \%$ trichloroacetic acid was added, and the mixture was centrifuged at $3000 \mathrm{r} / \mathrm{min}$ for $15 \mathrm{~min}$. The volumes of $40 \mu \mathrm{L}$ supernatant liquor were deter- mined using high-performance liquid chromatography. The ATFX was separated on $125 \times 4 \mathrm{~mm}$ LiChroCART125-4 column (Merk KGaA Company) with $5 \mu \mathrm{m}$ particles, and the mobile phase consisted of a mixture of acetonitrile $/ 0.05 \mathrm{~mol} / \mathrm{L}$ potassium dihydrogen phosphate buffer ( $\mathrm{pH} 3.0,13: 87$, volume/ volume) with a flow rate of $0.8 \mathrm{~mL} / \mathrm{min}$. The wavelength of UV-Vis detector was $293 \mathrm{~nm}$ and the quantitation range was $0.029-7.5 \mathrm{mg} / \mathrm{L}$. The standard curve was $C=0.0531 \times S+0.0148$ with the correlation coefficient $r=0.998$, where $C$ was the sample concentration and $S$ was the determined concentration. The linear range of the standard curve was $0.029-7.50 \mathrm{mg} / \mathrm{L}$ and the lower limit of quantification was $0.029 \mathrm{mg} / \mathrm{L}$. The coefficients of variation and relative standard deviation (RSD) were 0.010 and $1.09 \%$ (intra-day), respectively, and $0.015 \%$ and $1.47 \%$ (inter-day) for the range of $0.029-7.50 \mathrm{mg} / \mathrm{L}$.

\section{Population pharmacokinetic analysis}

The time-concentration data of multiple dose design were collected, and PPK analysis was conducted using the nonlinear mixed effects modeling program (NONMEM, Version 7.2, ICON Development Solutions, USA) with the interface Wings for NONMEM to estimate the population parameters, interindividual variability, and intraindividual variability. The Stepwise Covariate Model (SCM) module in Perl Speak to NONMEM software (ver 3.2.4, Uppsala University, Sweden) ${ }^{[10]}$ was applied for covariate screening and identification. The prediction-corrected visual predictive checks ( $\mathrm{pcVPC}$ ) were simulated by Perl Speak to NONMEM and plotted by Xpose (version 4.0, Uppsala University, Sweden). The interindividual variability for each structural parameter of the basic model was modeled using the exponential error model:

$$
P_{\mathrm{i}}=P_{\mathrm{TV}} \times \operatorname{Exp}\left(\eta_{\mathrm{i}}\right)
$$

Where $P_{\mathrm{i}}$ is a parameter in an ith individual, $P_{\mathrm{TV}}$ is a typical value of the parameter, and $\eta_{\mathrm{i}}$ is a random variable with a zero mean and a variance of $\omega^{2}$. The residual variability was evaluated (include additive error, proportional error, combined additive model) to describe the intraindividual variability. The combined additive and proportional error model based on the following equation best described the residual variability:

$$
C_{\mathrm{ij}}=C_{\text {pred, }, \mathrm{ij}} \times\left(1+\varepsilon_{\text {prop, ij }}\right)+\varepsilon_{\text {add, ij }}
$$

Where $C_{\mathrm{ij}}$ is the jth observed value in the ith subject, $C_{\text {pred, }, \mathrm{ij}}$ is the jth predicted value in the ith subject, and $\varepsilon_{\text {prop, ij }}$ and $\varepsilon_{\text {add, ij }}$ are the residual intraindividual variability with means of zero and variances of ${\sigma_{\text {pro }}}^{2}$ and $\sigma_{\text {add }}{ }^{2}$, respectively. The PPK model was estimated using the first-order conditional estimation (FOCE) method with $\eta-\varepsilon$ interaction.

The base model was selected on the basis of fit statistics (likelihood ratio test), residual analysis and parameters rationality. The criteria of statistical significance are determined by a decrease in the objective function value (OFV) greater than $3.84(P<0.05, \mathrm{DF}=1)$ or $5.99(P<0.05, \mathrm{DF}=2)$. For volunteers who received doses in a different sequence, $\operatorname{IOV}^{[11]}$ was taken into account for random effects modeling.

\section{Building the covariate model}

The aim of covariate analysis is to identify any covariates that 
explain a significant portion of individual difference. Linear function was used to incorporate continuous covariates into the model as follows:

$$
\mathrm{P}_{\mathrm{TV}}=\theta_{1} \times\left[1+\theta_{2}\left(\mathrm{COV}-\mathrm{COV}_{\text {median }}\right)\right]
$$

where $\theta_{1}$ represents the parameter estimate for an individual with COV equal to its median, and $\theta_{2}$ represents the change in the parameter estimate associated with the difference between covariates from its median.

In the case of categorical covariate, the introduction of ways was a piece-wise model as follows:

$$
P_{\mathrm{TV}}= \begin{cases}\theta_{1} & \text { if } \mathrm{COV}=0 \\ \theta_{2} \times\left(1+\theta_{2}\right) & \text { if } \mathrm{COV}=1\end{cases}
$$

where $\theta_{1}$ represents the parameter of the subjects with the covariate categorized as 0 , and $\theta_{2}$ represents the difference between the categories. The categorical covariate sex $(\operatorname{sex}=0$, female; sex $=1$, male) is binary variable in the study.

The stepwise approach of adding the covariate was applied to the model building, with the evaluation of each step dependent on the changes in the OFV. The statistical criteria for a covariate to be retained in the model were a decrease in the OFV greater than $6.63\left(P<0.01, \chi^{2}\right.$ distribution with one degree of freedom) during forward selection and an increase in the OFV less than $7.88(P<0.05)$ during backward elimination.

\section{Approach of model evaluation and validation}

The bootstrap option for assessing the stability and performance of the model was used to carry out the nonparametric bootstrap using the Wings for NONMEM software package. Repeated random sampling generated one thousand replicates with replacement from the original dataset. The final model's parameter estimates and its 95\% confidence intervals were compared to the median and the 2.5th and the 97.5th percentiles of the 1000 bootstrap results.

The pcVPC was used as an informative diagnostic tool to examine model appropriateness over time as well as covariate values $^{[12]}$ and was implemented by Xpose. ATFX concentration profiles were simulated 1000 times and compared with observed data to evaluate the predictive performance of the model.

\section{Determination of MIC in vitro}

Escherichia coli, Staphylococcus aureus, Klebsiella pneumonia, Staphylococcus aureus, and Staphylococcus epidermidis were the bacterial strains analyzed. The MICs of each bacteria in vitro, illustrated in Table 1, were extracted from previous pharmacodynamics research, in which they were obtained

Table 1. MIC determined in four bacteria.

\begin{tabular}{lc}
\hline Bacteria Strain (№ of strain) & MIC (mg/L) \\
\hline Escherichia coli (33) & 1.000 \\
Klebsiella pneumonia (30) & 0.125 \\
Staphylococcus aureus (38) & 0.250 \\
Staphylococcus epidermidis (22) & 0.250 \\
\hline
\end{tabular}

using the agar-dilution sensitivity test ${ }^{[13]}$ and disc-diffusion sensitivity test ${ }^{[14]}$.

\section{Dose regimen selection and validation method}

As described in the PPK model, it is possible to predict the relationship between time and serum concentration after receiving a dose. The AUC within the five days was simulated after 200, 300, and $400 \mathrm{mg} / \mathrm{d}$ dose administration, and 200, 300 , and $400 \mathrm{mg} / \mathrm{d}$ dose administration following a loading dose of 400,600, and $800 \mathrm{mg} / \mathrm{d}$, respectively. The ratios of AUC/MIC with and without a loading dose were calculated.

Although some research suggests that AUC/MIC should be over 30 when treating Streptococcus pneumoniae infections ${ }^{[15]}$, and more than 100 to achieve a sufficient clinical cure when treating other infections ${ }^{[16,17]}$, the aim of our study is dose finding for an early exploratory trial. Therefore, in our study, the AUC/MIC was set to more than 20, and the performance of dose regimen was evaluated using a phase II clinical trial.

\section{Results}

\section{Demographic information}

There were twenty-four volunteers (12 male and 12 female) enrolled in our study, and their demographic characteristics and general data are shown in Table 2.

Table 2. Demographics of subjects enrolled. $n=24$. Mean $\pm S D$.

\begin{tabular}{lcc}
\hline & Mean \pm SD & Range \\
\hline Age (year) & $26.00 \pm 4.41$ & $20-35$ \\
Height $(\mathrm{cm})$ & $166.42 \pm 7.47$ & $151-178$ \\
Weight $(\mathrm{kg})$ & $58.58 \pm 6.51$ & $50-77$ \\
Body mass index & $21.17 \pm 1.76$ & $18-25$ \\
Sex (male/female) & $12 / 12$ & \\
\hline
\end{tabular}

\section{Concentration-time profiles of ATFX}

A total of 24 subjects received multiple doses of 200 and 400 mg during the two periods. The mean concentration-time profiles on the fifth day of each period are shown in Figure 1. Compartment models with and without absorption lag time (ALAG) were tested for basic model fitting.

\section{Basic model}

A three-compartment model failed in regards to the timeconcentration data, and a two-compartment model was successful without ALAG and was better than one-compartment model $(P<0.01)$. The modeling with first-order absorption was implemented with ADVAN4 and TRANS4 subroutines in NONMEM to estimate the parameter clearance (CL), volume distribution of central compartment $\left(V_{\mathrm{c}}\right)$, inter-compartmental clearance $(Q)$, volume distribution of the peripheral compartment $\left(V_{\mathrm{p}}\right)$, and absorption rate constant $\left(K_{\mathrm{A}}\right)$. The exponential error model and the combination error model was used to describe the interindividual variance and intraindividual vari- 


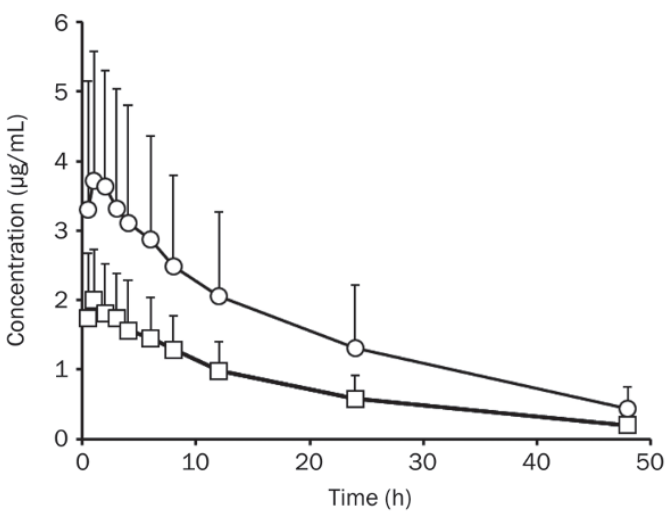

Figure 1. The concentration-time profiles in the fifth day for healthy volunteers after received $200 \mathrm{mg} / \mathrm{d}$ ( $\square$ ) and $400 \mathrm{mg} / \mathrm{d}$ (०) ATFX. $n=24$. Mean \pm SD.

ance, respectively. There was a significant reduction in the OFV after IOV was included in the model. In the basic model, the mean (RSE\%) population estimate values were $8.02(7.6 \%)$ for CL, $136(12.5 \%)$ for $V_{\mathrm{c}}, 14.3(31.3 \%)$ for $Q, 48.5(23.9 \%)$ for $V_{\mathrm{p}}$ and $2.82(20.7 \%)$ for $K_{\mathrm{A}}$.

\section{Final model}

The demographic and laboratory indexes were considered and introduced into the model, and changes in the OFV $(\Delta-2 \mathrm{LL})$ were investigated after each including and eliminat- ing step. After the stepwise covariate modeling, the weight as covariates on $V_{\mathrm{c}}$ and $\mathrm{CL}$, and the sex on $K_{\mathrm{A}}$ as covariate were retained in the final model. The final models were as follows:

$$
\begin{gathered}
K_{\mathrm{A}}=\theta_{2} \times K_{\mathrm{A}} \mathrm{COV}, K_{\mathrm{A}} \mathrm{COV}= \begin{cases}1 & \text { if } \mathrm{SEX}=1 \\
1+\theta_{\text {SEX_KA }} & \text { if SEX }=0\end{cases} \\
\mathrm{CL}=\theta_{1} \times\left[1+\theta_{\text {WEIGHT_CL }} \times(\text { WEIGHT-59.00) }]\right. \\
\mathrm{V}_{\mathrm{c}}=\theta_{2} \times\left[1+\theta_{\text {WEIGHT_V1 }} \times(\text { WEIGHT-59.00) }]\right.
\end{gathered}
$$

where $\theta_{1}, \theta_{2}$, and $\theta_{3}$ are the population parameter of $C L, V_{c}$, and $K_{\mathrm{A}}$, respectively, and 59.00 is the median of body weight. $\mathrm{SEX}=1$ represents male and SEX $=0$ female in $K_{\mathrm{A}} \mathrm{COV}$. The inclusion of interoccasion variability caused a large reduction in the OFV, and it was decided to evaluate the covariates with the IOV included in the model.

The population parameter estimates of the two-compartment model with an IOV for $\mathrm{CL}, V_{\mathrm{c}}, Q$, and $V_{\mathrm{p}}$ are illustrated in Table 3. After weight and sex were included in the final model as covariates, the estimates were $8.34(6.4 \%), 142$ (10.4\%), 15.9 (38.3\%), $52.2(20.1 \%)$, and $4.64(30.8 \%)$ for CL, $V_{c^{\prime}}$ $Q, V_{\mathrm{p}}$, and $K_{\mathrm{A}}$, respectively. The omission of the covariates from $\mathrm{CL}, K_{\mathrm{A}}$, and $V_{\mathrm{c}}$ increased the OFV by 516.5, 514.9, and 506.0, respectively. This clearly indicated that inclusion of weight and sex improved the fit.

The final model was tested by nonparametric bootstrap, and the results demonstrated a complete overlap of the 95\% confidence interval of parameter estimates with the ranges of the 2.5th to 97.5 th percentiles, indicating that the final model

\begin{tabular}{|c|c|c|c|c|}
\hline Parameter & \multicolumn{2}{|c|}{ Final model } & \multicolumn{2}{|c|}{ Bootstrap } \\
\hline \multicolumn{5}{|l|}{ PK Parameter } \\
\hline $\mathrm{CL}(\mathrm{L} / \mathrm{h}), \theta_{1}$ & $8.34(6.4)$ & $7.301-9.379$ & 8.39 & $7.46-9.71$ \\
\hline$V_{c}(L), \theta 2$ & $142(10.4)$ & $113.2-170.8$ & 135 & $66.6-173$ \\
\hline$K_{A}(/ h)$ in male, $\theta 3$ & $4.64(30.8)$ & $1.837-7.443$ & 4.34 & $1.48-11.9$ \\
\hline$\theta_{\text {WEIGHT_CL }}$ & $0.035(23.8)$ & $0.019-0.051$ & 0.0356 & $0.021-0.053$ \\
\hline$\theta_{\text {WEIGHT_Vc }}$ & $0.055(21.2)$ & $0.032-0.077$ & 0.0553 & $0.030-0.110$ \\
\hline$\theta_{\text {SEX_KA }}$ & $-0.629(16.7)$ & $-0.835--0.423$ & -0.654 & $-0.860--0.352$ \\
\hline$K_{\mathrm{A}}, \%$ & $33.2(57.3)$ & & 20.0 & $0.332-68.5$ \\
\hline IOV for $C L, \%$ & $19.1(17.4)$ & & 19.0 & $12.7-25.4$ \\
\hline IOV for $V_{c}, \%$ & $41.1(23)$ & & 41.9 & $15.7-79.2$ \\
\hline \multicolumn{5}{|l|}{ Residual variability } \\
\hline Proportional error, \% & $31.03(18.6)$ & & 30.7 & $25.3-37.1$ \\
\hline Additive error ( $\mu \mathrm{g} / \mathrm{mL})$ & $9.71(60.1)$ & & 9.30 & $2.66-16.8$ \\
\hline
\end{tabular}
was robust (Table 3). The final model demonstrated strong

Table 3. Parameter estimates, standard error, and bootstrap confidence intervals of the final model.

SEX $=0$, female; $S E X=1$, male. 
stability, with 980 out of 1000 bootstrap runs fitting successfully, and bootstrap estimates very similar to the population estimates.

\section{Model validation and evaluation}

The goodness of fit for final model illustrated the good correlations of the population predictive value (PRED), individual predictive value (IPRED) and observed value (OBS) and their closeness to the trend line and identity line (Figure 2), and the conditional weighted residuals (CWRES) value distributed evenly around zero with most of the points located between \pm 4 . Figure 3 shows the results of the pcVPC using 1000 Monte Carlo simulations. The observed concentration was included in the range of confidence intervals, and the median and 95\% confidence interval lines were located near the middle of the 1000 results, suggesting the sufficiency of the model's predictive power.
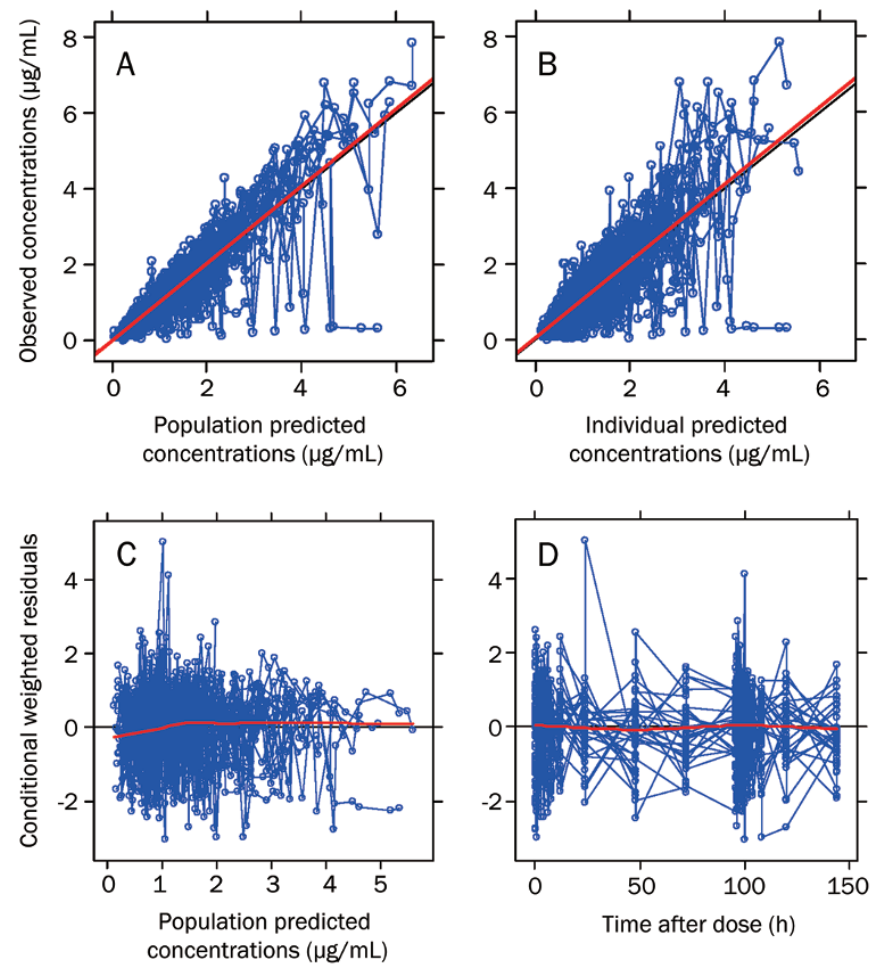

Figure 2. Final model validation. (A) Population predicted concentration versus observed concentration. (B) Individual predicted concentration versus observed concentration. (C) Conditional weighted residuals versus population predicted concentration. (D) Conditional weighted residuals versus time. The black line and red line in $(A)$ and $(B)$ represent the line of identity and regression line, respectively, whereas in (C) and (D) are the position where conditional weighted residual equal 0 and the red lines are the nonparametric regression line.

\section{Parameters for dose finding}

The concentrations of ATFX with and without a loading dose were calculated according to the final model. Figure 4
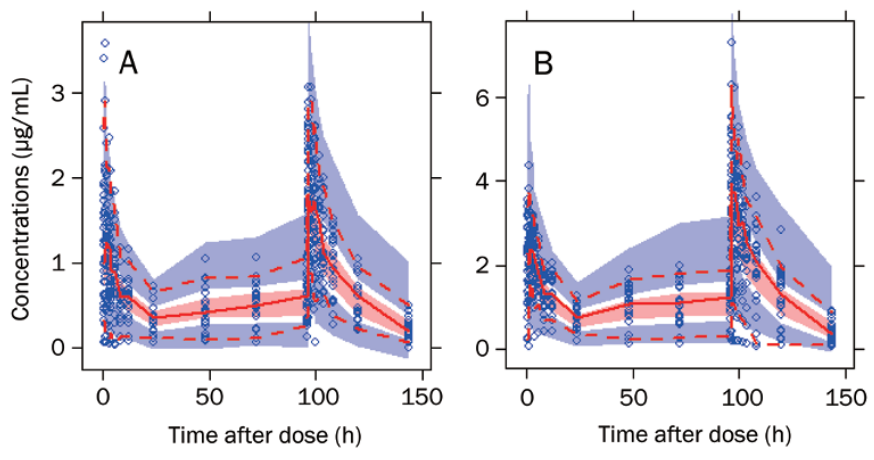

Figure 3. The prediction corrected visual predictive checks for the final model in dose regimens of (A) $200 \mathrm{mg} / \mathrm{d}$ and (B) $400 \mathrm{mg} / \mathrm{d}$. Open circles represent observed ATFX concentrations, the solid line and the dashed line represents the median and the $95 \% \mathrm{Cl}$ of observation, respectively. The middle red shadow areas represent the 95\% confidence intervals of median for the results of 1000 times simulation of final model and the blue shadow areas represent the $95 \%$ confidence intervals of the 2.5 th and 97.5 th percentiles of the results of 1000 times simulation of final model.

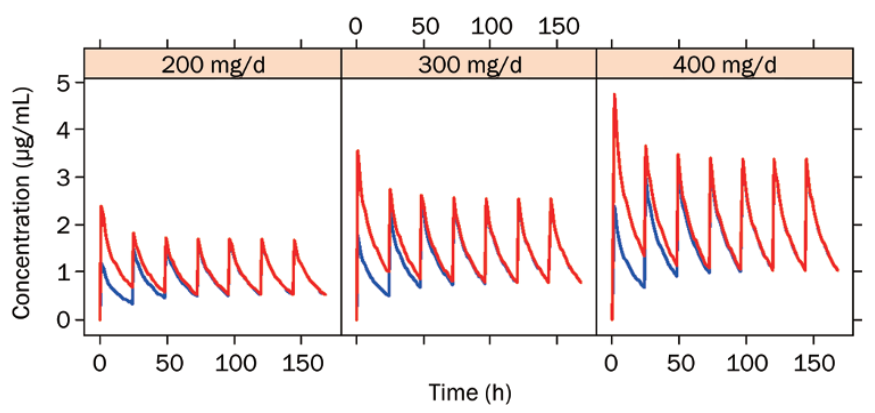

Figure 4. The simulated concentration-time curves for $7 \mathrm{~d}$ in healthy volunteers after they received multiple dose $(200,300$, and $400 \mathrm{mg} / \mathrm{d}$, respectively). The blue lines represent conventional designs and the red ones represent designs with loading dose.

describes the concentration-time profiles. In the case of conventional dose regimens, the ATFX blood concentration would be close to a steady state on the third day after the first dose received. However, the concentration with a loading dose would exceed the steady state immediately with the first dose.

The bacterium efficacy indicator displayed in Figure 5 indicates AUC/MIC to be affected by drug concentration and MIC simultaneously. The comparisons between the 4 types of bacterium revealed the greatest efficacy in Klebsiella pneumonia and the least in Escherichia coli. This finding is consistent with bacterial sensitivity. The AUC/MIC was close to a steady state on the third day with an identical dose, whereas a steady state was exceeded promptly after the administration of a loading dose.

The ATFX MIC against four types of bacteria ranging from 0.125 to $1 \mathrm{mg} / \mathrm{L}$ showed Klebsiella pneumonia had the highest sensitivity to ATFX, while Escherichia coli had a relatively lower sensitivity. According to the MIC and the $\mathrm{AUC}_{0-24}$ 


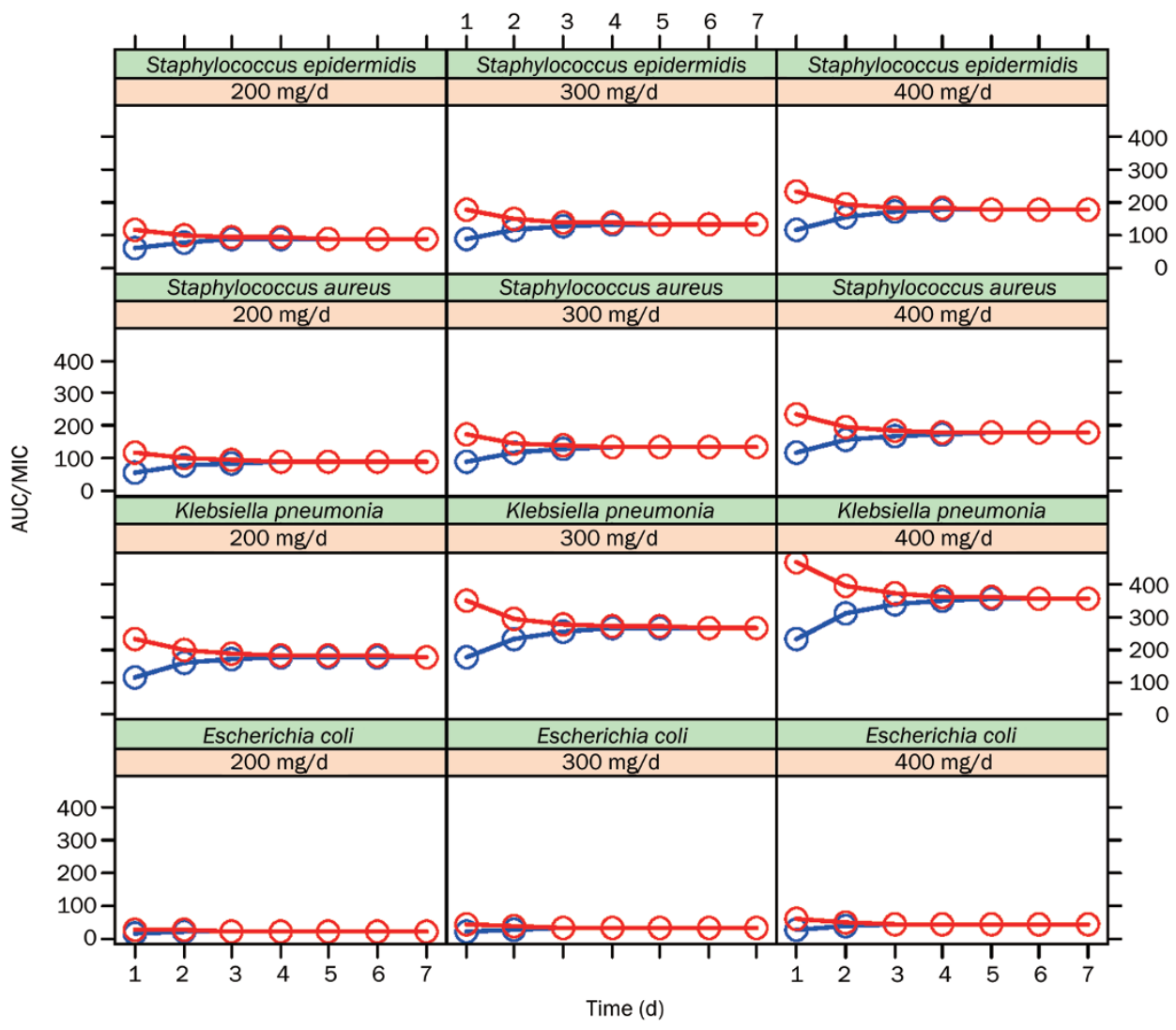

Figure 5. The simulated AUC/MIC-time profile of ATFX in four types of bacterium. The red lines represent the loading dose design and the blue lines represent the conventional design. calculated using a trapezoidal method, AUC/MIC was higher during a dose increase and exceeded the steady AUC/MIC immediately in the case of a loading dose design (Figure 5). AUC/MIC consistently approached a stable state after the third day with the change of blood concentration in multiple dose regimens.

\section{Dose regimen and its validation in phase II study}

Each value of AUC/MIC was more than 20 with $400 \mathrm{mg} / \mathrm{d}$ as a loading dose and $200 \mathrm{mg} / \mathrm{d}$ as a maintenance dose for treating infections. This dose regimen was recommended for the phase II trial and it demonstrated good efficacy in the treatment of acute pyelonephritis (AP) and acute exacerbations of chronic bronchitis (AECB) compared to levofloxacin ${ }^{[18]}$ (Table 4).

Table 4. Bacterial efficacies of three pathogens in ATFX phase II clinical trial.

\begin{tabular}{lcc}
\hline Pathogens of infections & Antofloxacin & Levofloxacin \\
\hline Escherichia coli & $30 / 30$ & $33 / 36$ \\
Klebsiella pneumonia & $27 / 28$ & $16 / 19$ \\
Staphylococcus spp & $5 / 5$ & $7 / 7$ \\
\hline
\end{tabular}

\section{Discussion}

According to the annual sales of antibiotics in China, fluoro- quinolones occupied the first or second class of antibacterial agents in Chinese market ${ }^{[19]}$. As a concentration-dependent antibiotic, the efficacy of ATFX can largely be reflected and predicted by drug concentration. However, little is known about the effect of the variance of the PK of ATFX among different individuals. The PPK model established in our study evaluated the influence of covariates on pharmacokinetic parameters quantitatively, and the application of the PPK study will be useful for personalized therapy.

The pharmacokinetics of ATFX in healthy male and female Chinese subjects can be described significantly better by a twocompartment model. The population estimates were similar to those of previous research ${ }^{[4]}$. We compared the effect of volunteers' demographic information and the population analysis revealed the body weight had significant influence on clearance rate and volume of distribution in the central compartment, while sex is the major covariate which affecting the absorption rate. The subjects with higher body weight may have a higher volume distribution and clearance, but the cause of $K_{\mathrm{A}}$ affected by sex is not clear. The sample size for this study was small, and therefore, these covariates should be evaluated in larger trials.

Concentration-dependent antibiotics with a low AUC/MIC may be reasonable for dose finding that can be applied to different types of infections. Otherwise, due to its low sensitivity to ATFX, Escherichia coli might be excluded from further clinical trials. Although the dose regimen was applied in a phase II clinical trial without consideration of individual character- 
istics such as body weight and sex, it still showed satisfactory efficacy and safety. However, further individual therapy for patients should be observed according to the covariates in a larger scale trial.

There are still some limitations in our study, as it is based on healthy volunteers. The pharmacokinetic features in children, the elderly and patients with infections or renal or hepatic disorders require further investigation. Moreover, the QT interval prolongation effect is a recognized adverse effect associated with many fluoroquinolones ${ }^{[20]}$, and the influence of ATFX on QT interval is not yet clear.

In conclusion, ATFX in healthy Chinese volunteers can be best described by a two-compartment model, and the patients' weight and gender may significantly affect the pharmacokinetic features. Based on the ratio of AUC from the PPK model and the MIC, the dose regimen we recommended is effective for treating bacterial infections. The application of population pharmacokinetics in dose finding can serve as a reference for personalized therapy with covariates in future research.

\section{Acknowledgements}

This work is supported by the Program for Shanghai Innovative Research Team in University (2009), the E-institutes of the Shanghai Municipal Education Commission (E03008) and the National Science and Technology Supporting Projects of China (2008BAI51B03).

\section{Author contribution}

Qing-shan ZHENG designed the research and revised the paper; Yun-fei LI and Kun WANG performed the research; Yun-fei LI wrote the paper; Fang YIN, Ying-chun HE and Jihan HUANG helped analyze data.

\section{References}

$1 \mathrm{Ye} \mathrm{H}, \mathrm{Wu}$ J, Yang Y, Chen K, Ji R. Antibacterial activities of the derivatives-YH54 and YH57 of levofloxacin in vitro. Chin Pharmacol Bull 2002; 18: 112-13.

2 Food and Drug Administration. Guidiance for industry: Population pharmacokinetics. U.S. department of healthy and human service 1999.

3 Lu Y, Kang ZS, Zhu Y, Zhang M, Liu Y, Li TY, et al. Pharmacokinetic study of single and multiple oral dose administration of antofloxacin hydrochloride in healthy male volunteers. Chin Med J 2011; 124 : 242-5.

4 Wang J, Xiao Y, Lu Y, Kang Z, Zhang M, Liu Y, et al. Pharmacokinetics of antofloxacin hydrochloride in healthy male subjects after multiple intravenous dose administration. Xenobiotica 2011; 41: 561-6.
5 Xiao Y, Lu Y, Kang Z, Zhang M, Liu Y, Li T. Pharmacokinetics of antofloxacin hydrochloride, a new fluoroquinolone antibiotic, after single oral dose administration in Chinese healthy male volunteers. Biopharm Drug Dispos 2008; 29: 167-72.

6 Xiao Y, Lu Y, Kang Z, Hou F, Wang S, Li T, et al. Tolerability and pharmacokinetics of antofloxacin hydrochloride after multiple oral dose administration in healthy Chinese male volunteers. Int J Clin Pharmacol Ther 2008; 46: 172-9.

7 Craig WA. Pharmacokinetic/pharmacodynamic parameters: rationale for antibacterial dosing of mice and men. Clin Infect Dis 1998; 26 : 1-10; quiz 11-2.

8 Lister PD. Impact of AUC/MIC ratios on the pharmacodynamics of the des- $F(6)$ quinolone garenoxacin (BMS-284756) is similar to other fluoroquinolones. J Antimicrob Chemother 2003; 51: 199-202.

9 Hoang KD, Pollack CV Jr. Antibiotic use in the emergency department. IV: Single-dose therapy and parenteral-loading dose therapy. J Emerg Med 1996; 14: 619-28.

10 Jonsson EN, Karlsson MO. Automated covariate model building within NONMEM. Pharm Res 1998; 15: 1463-8.

11 Karlsson MO, Sheiner LB. The importance of modeling interoccasion variability in population pharmacokinetic analyses. J Pharmacokinet Biopharm 1993; 21: 735-50.

12 Bergstrand M, Hooker AC, Wallin JE, Karlsson MO. Prediction-corrected visual predictive checks for diagnosing nonlinear mixed-effects models. AAPS J 2011; 13: 143-51.

13 Xiao Y, Li Y, Liu J, Zhong W, Yang W. A primary study of antofloxacin breakpoints for agar-dilution sensitivity test. Chin J Antibiotics 2010; 35: 462-7. Chinese.

14 Xiao Y, Li Y, Liu J, Zhong W, Yang W. Primary study of antofloxacin breakpoints with disc-diffusion sensitivity test. Chin J Clin Pharmacol Ther 2010; 15: 298-304. Chinese.

15 Ambrose PG, Grasela DM, Grasela TH, Passarell J, Mayer HB, Pierce PF. Pharmacodynamics of fluoroquinolones against Streptococcus pneumoniae in patients with community-acquired respiratory tract infections. Antimicrob Agents Chemother 2001; 45: 2793-7.

16 Craig WA. Does the dose matter? Clin Infect Dis 2001; 33 (S3): S233-S37.

17 Forrest A, Nix DE, Ballow CH, Goss TF, Birmingham MC, Schentag JJ. Pharmacodynamics of intravenous ciprofloxacin in seriously ill patients. Antimicrob Agents Chemother 1993; 37: 1073-81.

18 Wang J, Xiao Y, Huang W, Xu N, Bai C, Xiu Q, et al. A phase II study of antofloxacin hydrochloride, a novel fluoroquinolone, for the treatment of acute bacterial infections. Chemotherapy 2010; 56: 378-85.

$19 \mathrm{Lin} \mathrm{H}$, Shen H. Antibiotics clinical usage in general hospital. Chin J Nosocomiol 2006; 16: 681-3.

20 Murphy ME, Singh KP, Laurenzi M, Brown M, Gillespie SH. Managing malaria in tuberculosis patients on fluoroquinolone-containing regimens: assessing the risk of QT prolongation. Int J Tuberc Lung Dis 2012; 16: 144-9. 